\title{
Optical hydrogen absorption consistent with a bow shock around the hot Jupiter HD 189733 b
}

\author{
P. Wilson Cauley ${ }^{1}$, Seth Redfield ${ }^{1}$, Adam G. Jensen ${ }^{2}$, Travis \\ Barman $^{3}$, Michael Endl ${ }^{4}$ and William D. Cochran ${ }^{4}$ \\ ${ }^{1}$ Van vleck Observatory, Wesleyan University, \\ 96 Foss Hill Dr., Middletown, CT 06457, USA \\ email: pcauley@wesleyan.edu \\ ${ }^{2}$ University of Nebraska-Kearney and Department of Physics \& Physical Science \\ 2401 11th Avenue, Kearney, NE 68849, USA \\ ${ }^{3}$ University of Arizona and Department of Planetary Sciences and Lunar and Planetary \\ Laboratory \\ 1629 E University Boulevard, Tucson, AZ 85721, USA
}

${ }^{4}$ The University of Texas at Austin and Department of Astronomy and McDonald Observatory 2515 Speedway, C1400, Austin, TX 78712, USA

\begin{abstract}
Hot Jupiters, i.e., Jupiter-mass planets with orbital semi major axes of $<10$ stellar radii, can interact strongly with their host stars. If the planet is moving supersonically through the stellar wind, a bow shock will form ahead of the planet where the planetary magnetosphere slams into the the stellar wind or where the planetary outflow and stellar wind meet. Here we present high resolution spectra of the hydrogen Balmer lines for a single transit of the hot Jupiter HD 189733 b. Transmission spectra of the Balmer lines show strong absorption $\sim 70$ minutes before the predicted optical transit, implying a significant column density of excited hydrogen orbiting ahead of the planet. We show that a simple geometric bow shock model is able to reproduce the important features of the absorption time series while simultaneously matching the line profile morphology. Our model suggests a large planetary magnetic field strength of $\sim 28$ G. Follow-up observations are needed to confirm the pre-transit signal and investigate any variability in the measurement.
\end{abstract}

\section{Introduction}

Hot planets, i.e., planets with orbital periods of a few days or less, can interact strongly with their host stars. Due to the large orbital velocities (on the order of $\sim 100 \mathrm{~km} \mathrm{~s}^{-1}$ ) of these planets, they can orbit supersonically through the stellar wind (Vidotto et al. 2010). The supersonic passage of the planet can result in a bow shock forming ahead of the planet in its orbit. If the planet has a magnetosphere, this bow shock can be magnetically mediated and detection of the bow shock can allow an estimate of the planetary magnetic field to be made. Due to the difficulty of detecting exoplanetary magnetic fields via radio emission (e.g., Murphy et al. 2015), this method of estimating field strengths may be a promising path forward.

Bow shocks forming ahead of hot planets will transit the host star before the disk of the planet, resulting in a pre-transit signal. Pre-transit absorption has been observed in a handful of hot Jupiter systems. The first detection was made by Fossati et al. (2010) who measured marginal absorption in the near-UV spectrum of WASP-12 b. This measurement was then modeled as a bow shock by Llama et al. (2011). Pre-transit absorption was detected in the HD 189733 b system by Ben-Jaffel \& Ballester (2013) 
and Bourrier et al. (2013) in a handful of UV metal lines. These measurements were also interpreted as being caused by a bow shock. Most recently, Ehrenreich et al. (2015) measured a strong pre-transit signal in Lyman- $\alpha$ around the hot Neptune GJ 436 b, although they find the absorption to be consistent with a large cloud of hydrogen rather than a bow shock geometry.

Here we present a strong pre-transit absorption signal detected in the Balmer lines of the hot Jupiter HD 189733 b. This is the most significant pre-transit detection to date. The short cadence of the observations allows us to see structure in the time series of the absorption, putting constraints on the geometry of the material causing the absorption.

\section{Observations and Data Reduction}

The observations were performed using HiRES (Vogt et al. 1994) on Keck I during the second half of the night on July 4, 2013. The B2 decker was employed, which has a slit size of $7.0^{\prime \prime} \times 0.57^{\prime \prime}$. This configuration resulted in a resolving power at $\mathrm{H} \alpha$ of $\sim 68,000$, or $4.4 \mathrm{~km} \mathrm{~s}^{-1}$. Individual exposure times were $3-5$ minutes. The signal-to-noise of the extracted spectrum at $\mathrm{H} \alpha, \mathrm{H} \beta$, and $\mathrm{H} \gamma$ was $\sim 400, \sim 180$, and $\sim 120$, respectively. The data were reduced using a package written by Jason X. Prochaska. All standard reduction steps were taken including bias subtraction, flat fielding, and the removal of cosmic rays and hot pixels. Wavelength solutions were performed using Th-Ar exposures taken at the beginning and end of the night. Telluric lines from the $\mathrm{H} \alpha$ order were removed by modeling the telluric absorption in an early-type telluric standard and then subtracting the scaled and shifted model from each spectrum. Telluric absorption in the $\mathrm{H} \beta$ and $\mathrm{H} \gamma$ orders is negligible.

\section{Transmission spectra}

The transmission spectrum is defined as

$$
S_{T}=\frac{F_{i}}{F_{\text {out }}}-1
$$

where $F_{i}$ is a single observation and $F_{\text {out }}$ is the master post-transit spectrum. Average transmission spectra for the Balmer lines $\mathrm{H} \alpha, \mathrm{H} \beta$, and $\mathrm{H} \gamma$ are shown in Figure 1 . The nine post-transit spectra are used as the comparison spectra (i.e., $F_{\text {out }}$ in eq. 3.1 ) to generate the transmission spectra. In order to test contribution of reduction systematics to the transmission signal (e.g., normalization or choice of comparison spectra), we perform an empirical Monte Carlo (EMC) procedure where the transmission spectrum is generated many thousands of times using different combinations of the comparison spectra. The resulting distributions give an estimate of the uncertainty in the absorption measurement. These distributions are shown in the fourth and fifth columns of Figure 1 for the inand pre-transit measurements, respectively. The blue distributions show comparisons between the spectrum of interest and combinations of the post-transit spectra; the green distributions show comparisons of the spectra of interest with themselves. All of the intransit absorption, which is caused by the extended atmosphere of the planet, is detected at $>3 \sigma$. The pre-transit absorption is detected at $>3 \sigma$ in both $\mathrm{H} \alpha$ and $\mathrm{H} \beta$ while being only marginally detected in $\mathrm{H} \gamma$.

Figure 2 shows the individual absorption measurements, i.e., the equivalent width of the transmission spectrum integrated from $-200 \mathrm{~km} \mathrm{~s}^{-1}$ to $+200 \mathrm{~km} \mathrm{~s}^{-1}$. The gap in the data from -110 minutes to -70 minutes was, unfortunately, used to take observations 


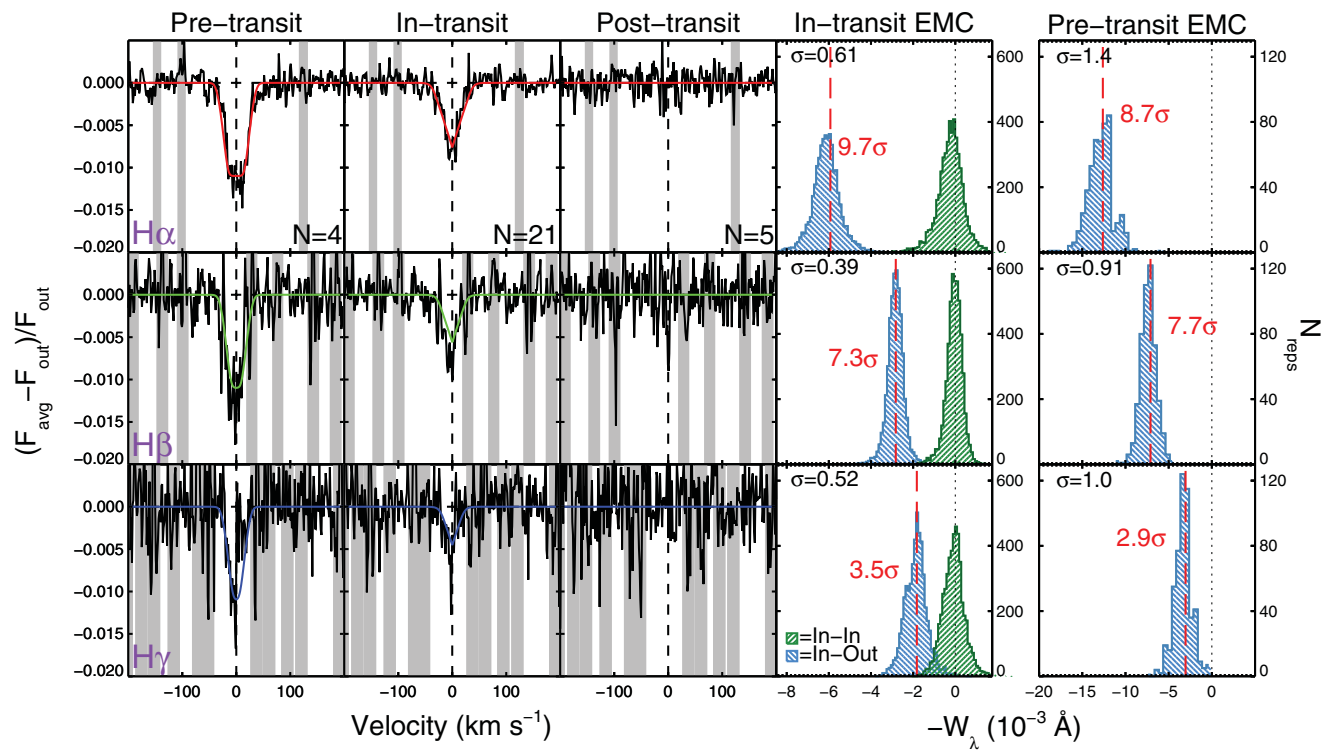

Figure 1. Transmission spectra of the Balmer lines for the pre-, in-, and post-transit epochs. Empirical Monte Carlo distributions are shown in the 4th and 5th columns for the in- and pre-transit epochs, respectively. The bow shock and exosphere model line profiles are shown as solid colored lines. Both the pre- and in-transit absorption is detected at a significant level for $\mathrm{H} \alpha$ and $\mathrm{H} \beta$. The pre-transit $\mathrm{H} \gamma$ absorption is marginally detected.

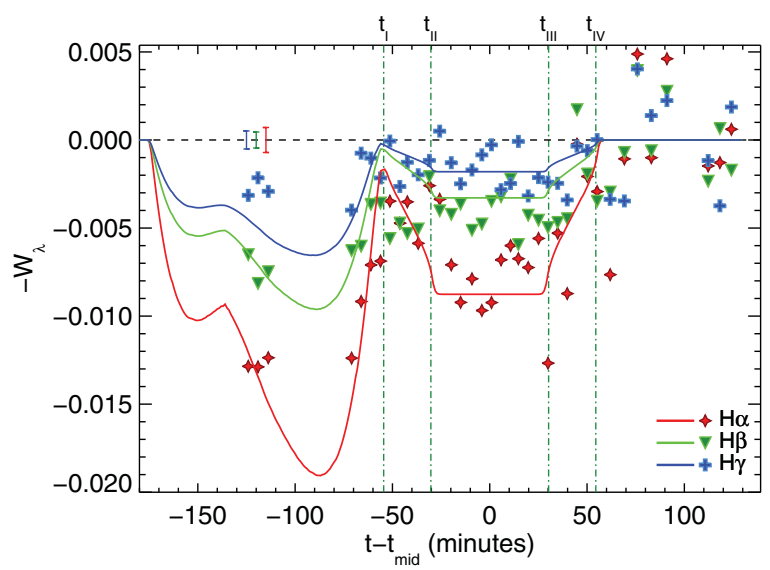

Figure 2. Time-series absorption for the Balmer lines. The bow shock-exosphere model is over plotted with solid colored lines. Representative uncertainties for the individual points are shown with the solid bars in the upper-left of the figure. Transit contact points are marked with vertical green lines and labeled at the top of the figure. Note the strong pre-transit absorption and the sharp decrease immediately before the optical transit.

of telluric standards, the pre-transit signal not being anticipated. Uncertainties derived from the EMC procedure for individual points are shown in the upper-left of Figure 2. The pre-transit absorption is very strong, $\sim 2$ times as strong, and shows a sharp decrease immediately before the optical transit of the planet. 

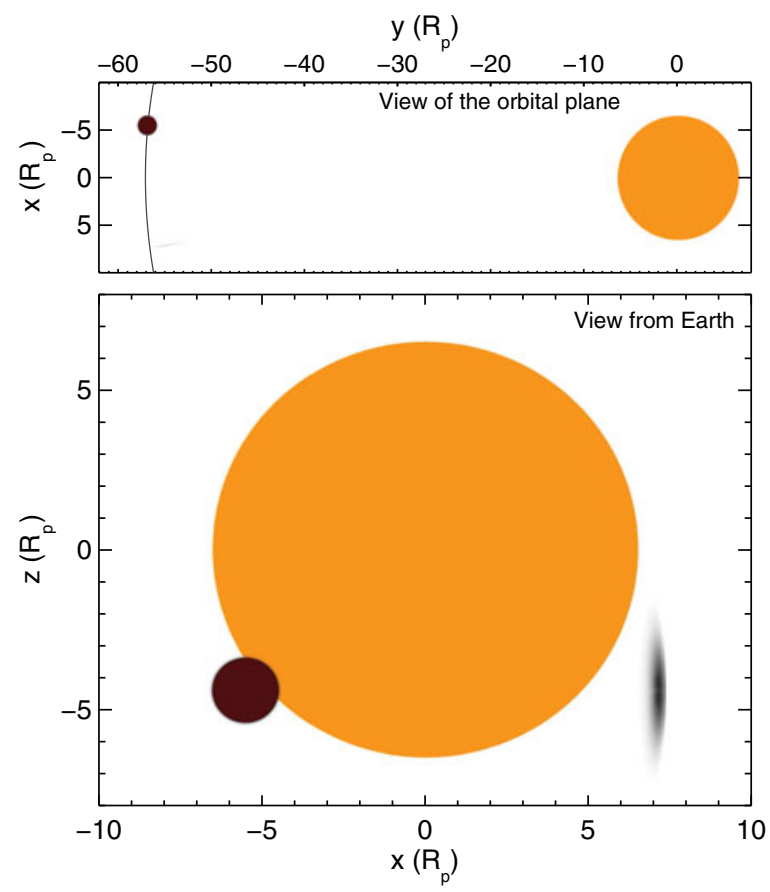

Figure 3. To-scale projections of the HD 189733 system with the model exosphere and bow shock. The top panel shows a slice of the orbital plane; the bottom panel shows a projection of the line-of-sight from Earth. The bow shock and exosphere are scaled to the same density. The exosphere can be seen as a small rim of absorbing material above the optical radius of the planet. The chosen snapshot at $t-t_{m i d}=-50$ minutes shows the time immediately after the bow has exited the disk and the planet is in between first and second contact.

\section{Bow shock model}

Motivated by the sharp decrease of the absorption immediately before the planetary transit, we modeled the absorption as arising in a narrow bow shock orbiting ahead of the planet. The sharp decrease of the absorption requires that the occulting structure quickly exit the stellar disk. A bow shock oriented perpendicular to the stellar surface can produce this type of absorption time series.

Using the analytic expression of Wilkin (1996) for the shape of the bow, we constructed a 3D bow shock and let it transit a uniform stellar disk. The model consists of a density at the nose of the bow $\rho_{0}$, a parameter $\alpha$ that determines how quickly the density decreases away from the nose, the angle between the nose and the planet's orbit $\theta_{0}$, and the standoff distance of the bow $r_{m}$. The line profiles are approximated using a Dopplerbroadened delta function. The thickness of the bow is $0.01 R_{p}$. We find this thickness to be necessary in order to match the absorption. We note that this is not an artifact of the grid resolution: setting the grid resolution to be $0.05 R_{p}$ still requires a bow thickness of $0.01 R_{p}$. However, anything larger than $0.01 R_{p}$ results in too much coverage of the stellar surface and subsequently too much absorption.

Our favored model is shown over plotted in Figure 2 with the solid colored lines. The resulting model line profiles are shown in Figure 1 with solid colored lines. A snapshot of the model through the $\mathrm{x}-\mathrm{y}$ and $\mathrm{x}-\mathrm{z}$ planes is shown in Figure 3. The model is able to approximately reproduce the important features of the absorption time series while simultaneously matching the line profile morphologies and line ratios. The model is computed using the parameter values $\theta_{0}=15^{\circ}, \rho_{0}=9 \times 10^{-20} \mathrm{~g} \mathrm{~cm}^{-3}, r_{m}=12.75 R_{p}$, and 
$\alpha=400$. Within the context of the model these parameters are fairly well constrained. For instance, changing $\theta_{0}$ to $40^{\circ}$ requires $r_{m}$ to be very large while simultaneously requiring $\alpha$ to be larger. Thus although there are degenerate solutions to the model, our favored parameters represent a compromise between a physically realistic system and a good match to the observations.

Since the angle at which the shock forms is determined by the relative velocities of the planet's motion and the stellar wind, the small value of $\theta_{0}$ found here suggests that the planet is moving through a slow region of the stellar wind or perhaps even a static corona. The standoff distance of $r_{m}=12.75 R_{p}$ is very large. If the bow shock is mediated by the planet's magnetosphere and we assume pressure balance between the stellar wind and the magnetosphere, this suggests a large equatorial magnetic field strength of $B_{e q}=28 \mathrm{G}$.

\section{Conclusions}

Pre-transit absorption has now been detected around a handful of hot exoplanets, suggesting it is a common phenomenon worthy of more intensive observations. These signals can arise from a rich variety of interactions between the planet and star. If the absorption is the result of enhanced densities in a bow shock mediated by the planet's magnetosphere, characterization and modeling of these signals could provide estimates of planetary magnetic field strengths, measurements which are otherwise very difficult to obtain. Our estimate of $28 \mathrm{G}$ for the magnetic field of HD $189733 \mathrm{~b}$ is very large and requires further investigation. More specifically, the contribution of stellar activity to the time series morphology needs to be more fully understood and other geometries (e.g., accretion streams from the planet) need to explored before estimates of exoplanetary field strengths can be well constrained.

\section{References}

Ben-Jaffel, L. \& Ballester, G. E. 2013, A\&A, 553, A52

Bourrier, V., Lecavelier des Etangs, A., Dupuy, H., et al. 2013,A\&A, 551, A63

Ehrenreich, D., Bourrier, V., Wheatley, P. J., et al. 2015, Nature, 522, 459

Fossati, L., Haswell, C. A., Froning, C. S., et al. 2010, ApJ, 714, L222

Llama, J., Wood, K., Jardine, M. et al., 2011, MNRAS, 416, L41

Llama, J., Vidotto, A. A., Jardine, M., et al. 2013, MNRAS, 436, 2179

Murphy, T., Bell, M. E., Kaplan, D. L, et al. MNRAS, 446, 2560

Vidotto, A. A., Jardine, M., \& Helling, Ch. 2010, ApJ, 722L, 168

Vogt, S. S., Allen, S. L., Bigelow, B. C., et al. 1994, SPIE, 2198, 362

Wilkin, F. P. 1996, ApJ, 459, L31

\section{Discussion}

FRANCE: What are you using in your model for the FUV input from the star?

CAUleY: The model is purely geometric, meaning that we are not solving for the physical conditions in the bow shock as a function of the system parameters (e.g., spectral type, strength of the stellar wind, etc.). We are assuming that some population of excited hydrogen exists with a bow-like geometry and seeing how much of this material and in what specific configuration it needs to be in in order to produce the observed absorption. A more complex (i.e., realistic) treatment is certainly warranted. 
UNKNOWN: How is the neutral hydrogen population maintained so far from the planet? Most models of hot Jupiter outflows show that the planetary material is almost completely ionized at large distances from the planet.

Cauley: It's possible that there is some recombination in the shock front due to the enhanced density. It's also possible that some form of charge exchange is occurring between the small neutral population from the planet and the protons from the stellar wind. This is an open question, however, and needs to be explored further. 